\title{
Current Pharmacists Condition in Pakistan and its Comparison with Foreign Pharmacists
}

\section{Muhammad Zeeshan Zafar}

Department of Pharmacy, University of Sargodha, Pakistan

*Corresponding author: Muhammad Zeeshan Zafar, Faculty of Pharmacy, University Of Sargodha, Pakistan, Tel: 03466189496; E-mail: shanmughal11@gmail.com

Received date: Sep 19, 2017; Accepted date: Sep 22, 2017; Published date: Sep 27, 2017

Copyright: () 2017 Zafar MZ. This is an open-access article distributed under the terms of the Creative Commons Attribution License, which permits unrestricted use, distribution, and reproduction in any medium, provided the original author and source are credited.

\section{Editorial}

Health is a broad concept that involves a multidisciplinary team of health care providers to deliver optimum health care to patients. Pharmacists are one of them whose major responsibilities are to manufacture medicines, assure their quality by quality control tests, dispense these dosage forms to right patients with counselling about health and medications, develop new dosage forms by researchers, and also prepare radiology pharmaceuticals for patient compliance [1-7].

Conditions of pharmacists in Pakistan concluded that pharmacist health care professionals are so neglected in Pakistan. There are more than $75 \%$ medical pharmacies which are running without qualified persons, without these persons how one can expect that he/she will receive right medications. In community setup of medications, pharmacists face low income and not any respect. On the other hand, if we see towards industrial and distributors of pharmaceuticals then desisted that a major reason for pharmacist's degradation is low income, work overload and insecurity of job. In Pakistan there are very few opportunities for pharmacists in the Government sector.

Pharmacist suggest to increase the pay for pharmacists in private as well as government sectors and request Government for announcing jobs for pharmacists in different healthcare fields. If government work for pharmacist's profession progress then their health facilities must increase, drug interactions decrease and increase the patient safety measures. Pharmacists also suggest about to make pharmaceutical care departments in all hospitals where pharmacists guide or counsel patients about their health and inform them about specific disease preventions.

It is sad to see that in some medical facilities, people with no degree in pharmacy are allowed to assume the position of pharmacists. This is not only extremely dangerous for patients but also unfair, because Pakistan is producing some great pharmacy graduates who are jobless. Where we take inspiration from Western countries to improve other sectors of our country, perhaps we should take heed and treat our pharmacy practitioners the way they treat theirs.

Moreover, pharmacists have the potential to ensure that patients have easy access to medication and medical expertise at all times, instead of waiting for physicians at hospitals to provide their undivided attention and discuss their medical charts. The Higher Education Commission of Pakistan needs to improve health plans at both state and private levels by making effort to strengthen the roots of pharmacy and health sciences. It is also a duty of pharmacists of Pakistan to work honestly for improving the health facilities in Pakistan and provide patient compliance.

By comparing Pakistani pharmacists with those of foreign countries, then cease that they treated pharmacists as trusted health professionals in advanced countries like U.K., U.S.A. etc. Foreign pharmacists have an important role in giving advice to patients for appropriate use of medicines, but pharmacists are the most neglected people in Pakistan. Pharmacists working abroad have huge opportunities for research work and a platform where they work freely to provide best health treatment to patients, but in Pakistan they can't follow these options. Pharmacists do not have any platform where they work and do research about new medications freely. Finally, pharmacists request to all health professionals to work for improving pharmacist's profession in Pakistan, because it's compulsory for better health.

\section{References}

1. http://www.who.int/tobacco/framework/final_text/en/index2.html

2. Hussain K (2010) Un-standardized and defective evaluation practices in the examination system in pharmacy institutes of Pakistan. Am J Pharm Educ 74: 16c.

3. Ghayur MN (2008) Pharmacy education in developing countries: Need for a change. Am J Pharm Educ 72: 94.

4. Azhar S, Hassali MA, Izham MI, Khan TM (2010) A qualitative evaluation of nurses' perception towards the role of pharmacist in healthcare setup in Pakistan. Health MED Journal 4: 71-76.

5. Anderson S (2002) Thestate of the World's pharmacy: A portrait of the pharmacy profession. Journal of Interprofessional Care 16: 391-404.

6. WHO (2006) Constitution Of The World Health Organization. Basic Documents 45: 1-18.

7. Khan $\mathrm{T}$ (2011) Challenges to pharmacy and pharmacy practice in Pakistan. The Australasian Medical Journal 4: 230-235. 